\title{
Asimetrías y consolidación progresiva de los intercambios académicos entre México y Japón, siglo XXI
}

\author{
Gerardo TANAmachi CaStro* \\ Sylvie Didou AupeTit**
}

* Maestro en Estudios de Asia y África en el Colegio de México.

** Investigadora del Centro de Investigación y Estudios Avanzados (CINVESTAV) del Instituto Politécnico Nacional y miembro de la Red sobre Internacionalización y Movilidades Académicas y Científicas (RIMAC), red temática del CONACYT.

\section{Resumen}

Este artículo presenta una panorámica de los intercambios universitarios entre Japón y México. Analiza los indicadores de movilidad estudiantil y convenios para identificar las asimetrías y las características de una cooperación académica emergente, simbólicamente importante pero todavía en ciernes en el ámbito de las IES.

\section{Palabras clave}

Cooperación académica; movilidad estudiantil; convenios; educación superior; México-Japón.

Assimetria e consolidação progressiva nos intercâmbios acadêmicos entre México e o Japão no século XXI

\section{Sumário}

Em esse artigo se apresenta uma panorâmica dos intercâmbios no nível superior entre o Japão e o México. Se analisam os convênios e os indicadores de mobilidade estudiantil para identificar as assimetrias e as características duma cooperação acadêmica emergente e simbolicamente importante, mas que ainda está começando no âmbito das IES.

Palavras-chave

Cooperação acadêmica; mobilidade estudiantil; convênios; educação no nível superior; México-Japão. 


\title{
Assymetries and continuing consolidation of the academic exchanges between Mexico and Japan in the XXI century
}

\begin{abstract}
This article presents a panorama of the university exchanges between Mexico and Japan. It analyzes the student mobility and conventions to identify the asymmetries and characteristics of an emerging academic cooperation, which is symbolically important, but still in process as part of the higher education institutions field.
\end{abstract}

\section{Key words}

Academic cooperation; Student mobility; Student conventions; Higher education; Mexico-Japan

\section{Introducción}

Las instituciones de educación superior (IES), en el mundo, experimentaron presiones para internacionalizarse, en las pasadas tres décadas (Vavrus \& Pekol, 2015: 5). Para responder a ellas, conformaron redes de transmisión o co-producción de conocimientos. Ejecutaron proyectos de diplomacia cultural para elevar la comprensión mutua entre culturas diferentes. Sobre todo, apoyaron la movilidad estudiantil.

Aunque el intercambio académico entre México y Japón no es significativo cuantitativamente para ninguna de las dos contrapartes, para México, esos vínculos académicos son de los más fuertes entre los que mantiene con una nación asiática. Igualmente, la relación con México es de las más importantes entre las que estableció Japón con los países de América Latina. En este artículo, consideraremos que ese intercambio es indisociable de la relación bilateral entre las dos naciones. Está influido por las políticas públicas, los recursos con los que cuentan los participantes, los acuerdos suscritos y los obstáculos acarreados por las asimetrías crecientes entre los niveles de desarrollo entre ambos países. En este orden de ideas, cabría preguntarse si cualquier cooperación académica entre un país más desarrollado (como Japón) y uno menos desarrollado (como México) genera desigualdad. 
Plantearemos que una modalidad sobresaliente de la internacionalización de la educación es la movilidad, entendida como el desplazamiento temporal de miembros de una comunidad académica a otra, con un propósito educativo específico (Quiroz, 2013: 59-60). Se lleva a cabo por iniciativas individuales o se rige conforme con lo dispuesto en los acuerdos bilaterales entre IES. Nuestra hipótesis es que la presión y los estímulos asociados a la internacionalización de las IEs en México y en Japón han redundado en la proliferación de acuerdos de intercambio académico. Sin embargo, las IES con más experiencia en la movilidad internacional tienen mayores posibilidades de aprovechar estos acuerdos. En los demás casos, los obstáculos económicos, políticos y culturales tienden a imponerse. Por otro lado, dado el respaldo que obtienen las IES japonesas del gobierno y del sector privado, su capacidad de negociación rebasa la de las mexicanas. Así, en México, los acuerdos son provechosos principalmente para la carrera profesional de los individuos participantes mientras que, en Japón, los beneficios se extienden al desarrollo institucional de las IES.

Presentaremos, en este artículo, un estado del arte de las investigaciones existentes en cada país sobre las movilidades internacionales, en general y entre México y Japón. Examinaremos el intercambio académico entre IES de ambos países, situándolo en el marco de su relación bilateral global. Identificaremos los recursos con los que cuentan los participantes y especificaremos los perfiles disímiles de los establecimientos académicos involucrados.

\section{La cooperación académica y la movilidad México-Japón: un tema con baja visibilidad}

Numerosas investigaciones han versado sobre la internacionalización de las IES. La Comparative and International Education Society, en 2014, último año en el que clasificó la bibliografía revisada por tema, reportó 73 referencias en el rubro "Internationalization and Study Abroad" (CIES, 2015: S2).

En Japón, la Japan Student Services Organization (JASSO) realiza estudios anuales sobre los estudiantes extranjeros en Japón y los japoneses en otros países. El Ministry of Education, Culture, Sports, Science 


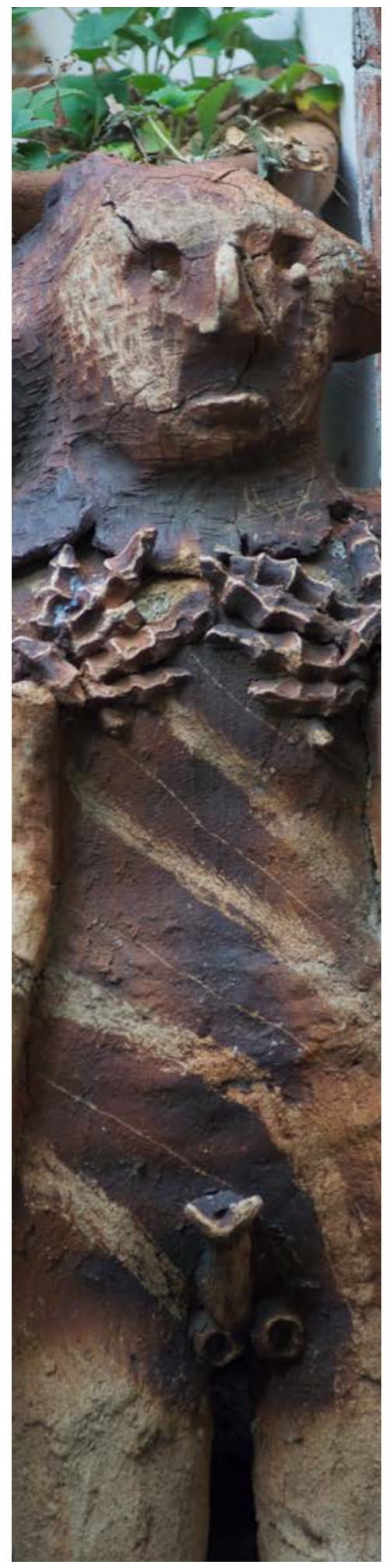

क्ष

26 Universidades $\mid$ núm. 84, abril-junio 2020 | UDUAL $\mid$ DOI:https://doi.org/10.36888/udual.universidades.2020.84.102

Gerardo Tanamacho Castro y Sylvie Didou Aupetit $\mid$ Asimetrías y consolidación progresiva de los intercambios académicos entre México y Japón, siglo XXI and Technology (MEXT) registra los acuerdos de movilidad académica suscritos por las IES japonesas. En la investigación académica, las revistas Hikaku Kyōikugaku Kenkyū, publicada por la Japan Comparative Education Society desde $1974,{ }^{1}$ y la Ibunkakan Kyöi$\mathrm{ku}$, editada desde 1987, por la Intercultural Education Society of Japan atienden con frecuencia el tema. Esa última dedicó sus números 5 (1991), 13 (1999) y 48 (2018) a los estudiantes en el extranjero. Por su parte, desde 2007, la Universidad de Kioto especializó su revista Kaigai Ryūgaku no Tebiki en los estudios en el extranjero. ${ }^{2}$ Entre 1955 y 2005, 800 textos concernieron a los estudiantes japoneses en el extranjero (Tsuboi, 2006). Más de la mitad data de los noventa. Versan, principalmente, sobre los japoneses en Estados Unidos y los asiáticos en Japón, los conflictos culturales y de comunicación y las políticas públicas. En los últimos quince años, las publicaciones sobre internacionalización abordaron los problemas de las universidades japonesas para internacionalizarse (Yonezawa, 2010), las dificultades para que aumente el número de japoneses que estudian en otros países (y el desequilibrio con el número de estudiantes extranjeros en Japón) (Ota, 2011) y los cambios en las políticas de internacionalización (Ota, 2012). Reflexionaron sobre la diversificación de los destinos y orígenes de los estudiantes (Rodríguez, 2013), la compatibilidad de los sistemas educativos de otros países con el de Japón (Shimauchi, 2014), el impacto a largo plazo de los estudios en el extranjero (Tanaka, 2010). Como temas supeditados, estudiaron el rol del lenguaje para la multiculturalidad y la internacionalización (Sugimura, 2015), el aumento de estancias muy cortas en el extranjero (Shinmi y Ota, 2018) y los debates sobre las habilidades que requieren los estudiantes para desempeñarse profesionalmente en un ambiente global (Abe, et al., 2018).

En México, la Asociación Nacional de Universidades e Instituciones de Educación Superior (ANUIES) coordinó dos encuestas de movilidad estudiantil internacional. ${ }^{3}$ García y Chapa (2016) integraron una revisión bibliográfica sobre las motivaciones de los estudiantes internacionales mexicanos al seleccionar una universidad destino. Además, estudios de caso sobre la internacionalización de las IES enfatizaron el papel de programas innovadores.

En contraste, la bibliografía sobre la movilidad estudiantil y la cooperación académica entre Japón y México es reducida. En México, Didou (1998) ana- 
lizó la cooperación científica y tecnológica japonesa en México y Barajas (2002) la técnica y cultural. Recientemente, otros estudios versaron sobre los servicios específicos que los japoneses consumen o contratan en las universidades mexicanas, tales como los cursos de español o las prácticas de vinculación con empresas (Didou, 2019: 163-164). Se hicieron estudios de caso sobre las IES mexicanas, públicas y privadas, que consolidaron su cooperación con Asia, estudiando las modalidades y experiencias de colaboración con Japón (Ramírez Bonilla, coord., 2017 y 2019). Finalmente, la Red sobre Movilidades Académicas y Científicas (RIMAC) subió a su página web entrevistas a especialistas de las relaciones México-Japón y a estudiantes mexicanos con experiencias de movilidad a dicho país. ${ }^{4}$ En Japón, Tanaka et al. iniciaron un proyecto etnográfico, en 2018, sobre las experiencias de los becarios japoneses en México, con soporte del Ministerio de Educación de Japón. Ochiai (2019) describió la cooperación entre la Universidad Hitotsubashi y el Colmex, ubicando su relevancia en la cooperación internacional de la primera institución.

En suma, aunque los temas de movilidad estudiantil y de internacionalización estén instalados en las agendas nacionales de investigación de ambos países, como tópicos generales, su visibilidad es mucho menor cuando están referidos a la relación bilateral entre México y Japón.

\section{La progresiva consolidación de un tejido de cooperación académica}

La historia de las relaciones diplomáticas y comerciales entre México y Japón repercutió siempre en las actividades académicas y científicas. El viaje a Yokohama en 1874 de una comisión mexicana para la observación del tránsito de Venus, encabezada por Francisco Díaz Covarrubias, fue un precedente de la firma, en 1888, del Tratado de Amistad, Comercio y Navegación entre ambos países, el cual fue el primer acuerdo suscrito por Japón en términos de igualdad (Toda, 2012). En 1897, inició la migración de japoneses a México, con respaldo gubernamental. Se establecieron en México escuelas para los hijos de los migrantes a cargo de profesores japoneses y fueron enviados a Japón estudiantes mexicanos de padres

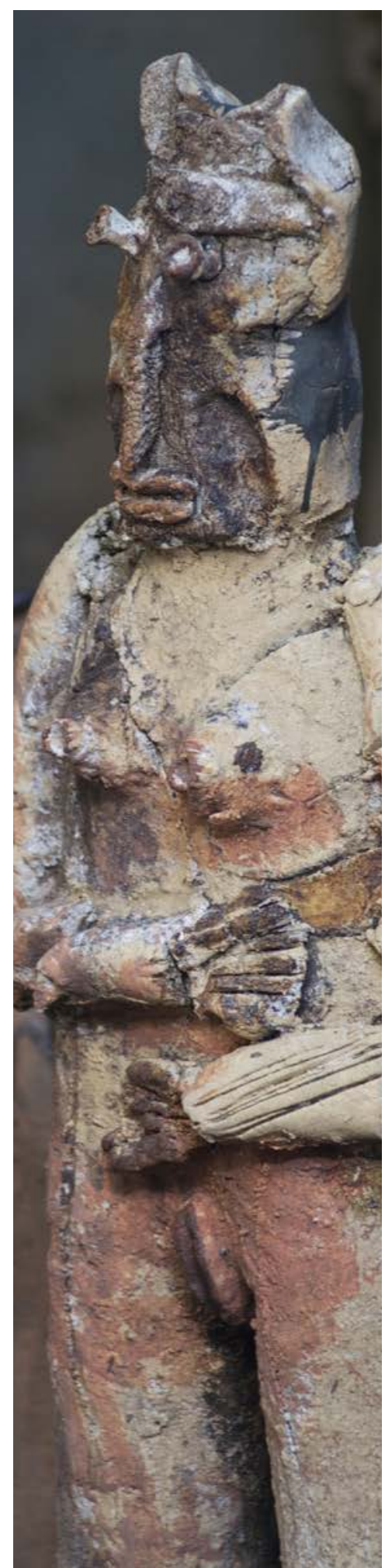


japoneses cuando ellos disponían de medios económicos suficientes (Ota Mishima, 1983: 35-93).

Las buenas relaciones entre México y Japón se mantuvieron a principios del siglo Xx. En 1917, los gobiernos de México y Japón firmaron el Convenio para el Libre Ejercicio de la Profesión de Médico, Farmacéutico, Dentista, Partero y Veterinario, vigente hasta 1928. Aunque ningún mexicano viajó a Japón al amparo de este acuerdo, decenas de especialistas japoneses ejercieron en México y transmitieron sus conocimientos a compatriotas suyos residentes en el país. ${ }^{5}$ El gobierno mexicano invitó en 1919 al bacteriólogo Hideyo Noguchi a Yucatán, para combatir la fiebre amarilla (Toda, 2013: 305-306). En los años siguientes, el biólogo Eizi Matuda y el director de teatro Seki Sano se mudaron a México (Toda, 2013: 147-148, 294-298). José Juan Tablada y Manuel Gamio visitaron Japón, así como seis estudiantes y un profesor de la Universidad Nacional Autónoma de México (UNAM) en 1930 (Uscanga, 2011a: 159-171).

La Segunda Guerra Mundial interrumpió esta dinámica, pues México le declaró la guerra a Japón en 1942 . No obstante, en los cincuenta, se reanudó con mayor fuerza. Aunque los vínculos entre ambos países se restablecieron de manera oficial hasta $1952,{ }^{6}$ en 1951, el físico Hideki Yukawa, galardonado con el premio Nobel en 1949, visitó México para asistir a los festejos de los 400 años de la fundación de la UNAM. ${ }^{7}$ En 1954, los gobiernos de México y Japón firmaron un convenio de cooperación cultural. ${ }^{8}$ Esa consolidación de la cooperación académica fue, además, concomitante a la expansión de la industria japonesa a nivel mundial. Empresas como Marubeni, Mitsui, Itochu, ${ }^{9}$ Telares Toyoda y Laboratorios Takeda se establecieron en México. En 1958, la Japan External Trade Organization (JETRO) instaló una oficina en este país (Toda, 2013: 198-211 y 229-236). Japón empezó a contribuir a la cooperación internacional, después de adherirse al plan Colombo ${ }^{10}$ en 1954 (Uscanga, 2011b: 85). Para coordinar los esfuerzos de la industria privada japonesa en dicha cooperación, en 1959, fundó la Association for Overseas Technical Cooperation and Sustainable Partnerships (AOTS), con el apoyo del Ministry of International Trade and Industry (MITI). ${ }^{11}$

Durante los sesenta, Mitsubishi, Sumitomo ${ }^{12}$ y Nissan invirtieron en México. La primera planta de producción que esta última empresa construyó fuera de Japón fue instalada en Cuernavaca (Toda, 2013: 217-228). A finales de la década, México se convirtió en beneficiario de las acciones de cooperación internacional de Japón (Embajada del Japón en México, 2016: 8) y ambos países firmaron un primer tratado comercial (Castañeda, 2000: 120). El Colegio de México (Colmex) y la UNAM crearon centros de estudios asiáticos. El Colmex contó con el apoyo del Proyecto Mayor Oriente-Occidente, patrocinado por la Organización de las Naciones Unidas para la Educación, la Ciencia y la Cultura (UNESCO) (Ota Mishima, 1975: 363-364).

En 1971, inició el Programa Especial de Intercambio para Estudiantes y Becarios Técnicos, bajo la responsabilidad del Consejo Nacional de Ciencia y Tecnología (CONACYT) y la Japan International Cooperation Agency ${ }^{13}$ (JICA). ${ }^{14}$ Originalmente con cien participantes de cada país al año, hasta la fecha, ha capacitado a más de 4,500 becarios. ${ }^{15}$ En los años siguientes, las relaciones entre ambos países se fueron diversificando. El MEXT de Japón empezó a asignar becas a mexicanos para estudios de posgrado (Castañe- 
da, 2000: 120). En 1977, el gobierno japonés donó alrededor de un millón de dólares para abrir el Liceo Mexicano Japonés (Toda, 2013:129-144). En 1980 se fundó la Asociación Japonesa de Estudios Latinoamericanos. ${ }^{16}$

Por su parte, en 1983, el gobierno japonés lanzó un plan para atraer a 100,000 estudiantes extranjeros en 2000. Sus objetivos eran fomentar la comprensión mutua con otras naciones, elevar el nivel educativo y de investigación de Japón y promover la formación de personal en los países en vías de desarrollo. ${ }^{17}$ En ese contexto, en 1986, México y Japón firmaron un convenio sobre cooperación técnica (Didou, 2019: 164), y, ese mismo año, Japón se convirtió en el segundo socio comercial de México después de Estados Unidos (Castañeda, 2000: 120). En 1987, la Fundación Japón estableció una oficina en México. ${ }^{18}$

Desde finales de los ochenta, México se involucró activamente en organismos internacionales. En 1989, ingresó como miembro oficial del Pacific Economic Cooperation Council (PECC). ${ }^{19}$ En 1994, firmó el Tratado de Libre Comercio de América del Norte (TLCAN), convirtiéndose en un atractivo receptor de inversión extranjera, por sus privilegios de exportación a Estados Unidos y Canadá. Ese mismo año, se incorporó al foro Asia-Pacific Economic Cooperation (APEC) ${ }^{20}$ (Ramírez, 2017: 13-14) y a la Organización para la Cooperación y el Desarrollo Económicos ${ }^{21}$ (OCDE). ${ }^{22}$

Ante este escenario, la Universidad de Guadalajara creó un Departamento de Estudios del Pacífico, y la de Colima un Centro Universitario de Estudios e Investigaciones sobre la Cuenca del Pacífico. Académicos de la UNAM y de El Colegio Mexiquense se sumaron a proyectos de investigación sobre dicha región del mundo, en la cual se ubica Japón (Ramírez, 2017: 14). En 1997, se formalizó el Esquema de Apoyo a la Cooperación Sur-Sur entre México y Japón, que incluía la instrumentación de proyectos de cooperación triangular (Uscanga, 2011b: 89).

Japón cumplió en 2003 con la meta de recibir 100,000 estudiantes extranjeros (Tsuboi, 2005: 1), y en 2008 lanzó un plan para hospedar a 300,000 en 2020. Este número representa aproximadamente el 10\% de la matricula inscrita en las universidades japonesas y equivale a alrededor del $5 \%$ de los estudiantes en movilidad internacional a nivel mundial. ${ }^{23}$

El gobierno japonés implementó programas para alcanzar ese nuevo objetivo, a través del MEXT y de su Japan Society for the Promotion of Science (JSPS). El primero fue el programa Global 30, lanzado en 2009, del 
que se beneficiaron 13 universidades japonesas. Su objetiFvo fue favorecer la internacionalización de las instituciones participantes con el desarrollo de programas académicos en inglés. ${ }^{24} \mathrm{El}$ año siguiente, el Inter-University Exchange Project (Re-Inventing Japan Project) promovió el intercambio académico con determinados países o regiones del mundo y en ciertas disciplinas. ${ }^{25}$

En 2012, comenzó el Project for Promotion of Global Human Resource Development, ${ }^{26}$ enfocado en la formación de recursos humanos capaces de enfrentar los retos de la globalización. A partir de 2014, este proyecto continuó en el programa llamado Go Global Japan. ${ }^{27}$ Ese mismo año, el programa Global 30 se transformó en el Top Global University Project ${ }^{28}$ e incluyó a 35 IEs japonesas. ${ }^{29}$

Por otro lado, la larga historia de las relaciones entre México y Japón fue una motivación para firmar, en 2004, el Acuerdo para el Fortalecimiento de la Asociación Económica entre México y Japón (Kerber, 2006: 356-373). Esta asociación se fortaleció cuando México y Japón suscribieron su Asociación Estratégica Global en 2010 (Granados, 2016: 80). Como resultado, actualmente, se encuentran establecidas en México más de 1,200 empresas japonesas. ${ }^{30}$

Para alentar la cooperación académica entre ambos países, desde 2011, se han llevado a cabo cuatro Cumbres de Rectores México-Japón. Éstas han propiciado la firma de acuerdos entre instituciones educativas y el fortalecimiento de las colaboraciones. En la primera de ellas (Tokio, 2011), los participantes recomendaron "fomentar la cooperación entre las sociedades del conocimiento y garantizar el intercambio continuo de puntos de vista en temas de interés común y la promoción de la educación, la ciencia y la innovación". ${ }^{31}$ En la segunda (Guanajuato y Aguascalientes, 2014), destacaron los vínculos establecidos entre instituciones mexicanas y la Universidad de Chiba. ${ }^{32}$ En la tercera (Hiroshima, 2017), discutieron principalmente la colaboración de triple hélice academia-industria-gobierno. ${ }^{33}$ En la Cuarta (Ciudad de México, 2019), atendieron tópicos como la Sociedad 5.0, la contribución de las universidades a la sustentabilidad, los riesgos y peligros relacionados con los fenómenos naturales y el futuro de las universidades. ${ }^{34}$

Así, a pesar de la distancia, del moderado número de estudiantes que circulan entre uno y otro país, de sus desigualdades económicas, de sus diferencias culturales y de sus sesgos en la estructuración de políticas públicas, la relación bilateral entre México y Japón es 
añeja y sólida. Recientemente, la importancia creciente de la industria japonesa en México ha confortado dicha tradición. Ahora, entre los beneficios esperados de la movilidad académica, no solo destacan la comprensión de una cultura distinta por parte de los participantes sino el mejoramiento de los perfiles profesionales y la creación de redes.

\section{El intercambio académico entre México y Japón: un flujo reducido}

Según la JASSO (2019a: 1), en 2000, 64,011 estudiantes extranjeros estaban inscritos en IES japonesas. Alrededor del 14\% lo hacía con algún tipo de beca del gobierno japonés y poco más del $2 \%$ recibía apoyo de su propio país. Más del $80 \%$ estudiaba con sus propios recursos. En 2018, dicha cifra llegó a 298,980 estudiantes, estando muy cerca de cumplirse la meta de los 300,000 estudiantes. Poco más del 3\% era becado por el gobierno japonés y alrededor del $1 \%$ por su propio país. Más del 95\% estudiaba con sus propios recursos. Del total, casi el 70\% estudiaba en IES y el resto en escuelas de idiomas. El 94.4\% de estos alumnos eran asiáticos y solo el $0.5 \%$ correspondía a latinoamericanos, entre ellos 315 mexicanos.

En contraste, el número de japoneses en el extranjero había disminuido: en 2010 , eran 60,000 pero, en 2014 , solo 54,000 (Ota \& Watabe, 2018: 21). Según el MEXT (2019: 4), la OCDE reportó, en 2016, 55,969 estudiantes japoneses en el extranjero. La JASSO (2019b: 6) informó que el año siguiente había 105,301, o sea, casi el doble. En las fuentes consultadas no se proporciona una explicación sobre dicha discrepancia en los datos. Según JASsO, cerca del 63\% del total viajó bajo las provisiones de algún acuerdo de intercambio académico. $63.5 \%$ hacia estancias menores a un mes. Alrededor del $37 \%$ estudiaba en el continente asiático y cerca del 27\% en Estados Unidos o Cana-

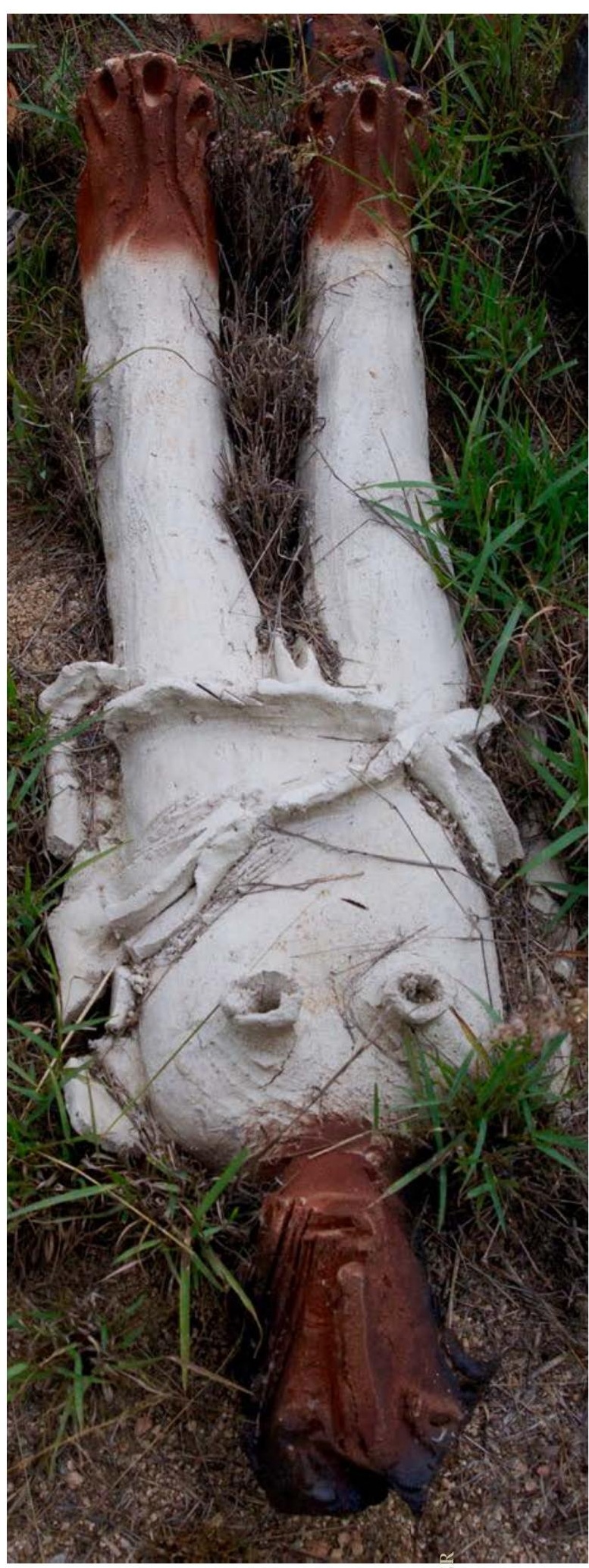

D
D
0
0 
dá. Los japoneses en América Latina ${ }^{35}$ correspondían a menos del 1\% del total.

En México, la encuesta Patlani de la ANUIES, en 2014-2015, registró 24,000 estudiantes en movilidad saliente y 15,608 en movilidad entrante y, en el ciclo siguiente, 29,401 y 20,322 respectivamente. En movilidad saliente, los principales países por orden de importancia fueron España, Estados Unidos y Francia. En movilidad entrante fueron Estados Unidos, Colombia y Francia (Maldonado, 2017: 17-31). Japón y México no fueron contrapartes significativas el uno para el otro.

Por otro lado, en su base de datos sobre los acuerdos bilaterales en los que participa Japón ${ }^{36}$ en 2016, el MEXT refiere 168 acuerdos entre IES japonesas y mexicanas. Involucran 88 instituciones japonesas (36 públicas, entre ellas 30 nacionales y 52 privadas) y 52 mexicanas (36 públicas y 16 privadas). 69 acuerdos corresponden a universidades públicas japonesas, 58 de ellas universidades nacionales y 99 privadas. 105 convenios fueron celebrados con instituciones públicas mexicanas y 63 con privadas.

La Tabla 1 muestra las once instituciones mexicanas y japonesas con mayor número de acuerdos. En México, se aprecia una concentración de universidades con acreditaciones nacionales e internacionales.

Tabla 1. Instituciones mexicanas y japonesas con más acuerdos

\begin{tabular}{|l|c|l|c|}
\hline \multicolumn{1}{|c|}{ Instituciones mexicanas } & $\begin{array}{c}\text { núm. de } \\
\text { acuerdos }\end{array}$ & \multicolumn{1}{|c|}{ Instituciones japonesas } & $\begin{array}{c}\text { núm. de } \\
\text { acuerdos }\end{array}$ \\
\hline $\begin{array}{l}\text { Universidad Nacional Autónoma de } \\
\text { México (UNAM) }\end{array}$ & 20 & Universidad de Chiba & 8 \\
\hline $\begin{array}{l}\text { Instituto Tecnológico y de Estudios } \\
\text { Superiores de Monterrey (ITESM) }\end{array}$ & 15 & $\begin{array}{l}\text { Universidad Tecnológica de } \\
\text { Nagaoka }\end{array}$ & 7 \\
\hline Universidad de Guanajuato (UGTO) & 13 & Universidad Sophia & 7 \\
\hline Universidad de Guadalajara (UdeG) & 11 & Universidad de Kansai Gaidai & 5 \\
\hline Universidad de Monterrey (UDEM) & 8 & Universidad Ritsumeikan & 5 \\
\hline Colegio de México (COLMEX) & 7 & Universidad Prefectural de Aichi & 4 \\
\hline $\begin{array}{l}\text { Universidad Autónoma de } \\
\text { Guadalajara (UAG) }\end{array}$ & 7 & $\begin{array}{l}\text { Universidad de Estudios } \\
\text { Internacionales de Kanda }\end{array}$ & 4 \\
\hline Universidad Iberoamericana (UIA) & 6 & Universidad de Kansai & 4 \\
\hline Instituto Politécnico Nacional (IPN) & 6 & $\begin{array}{l}\text { Universidad de Estudios } \\
\text { Extranjeros de Kioto }\end{array}$ & 4 \\
\hline $\begin{array}{l}\text { Benemérita Universidad Autónoma } \\
\text { de Puebla (BUAP) }\end{array}$ & 5 & $\begin{array}{l}\text { Universidad Internacional de } \\
\text { Osaka }\end{array}$ & 4 \\
\hline $\begin{array}{l}\text { Instituto Tecnológico Autónomo de } \\
\text { México (ITAM) }\end{array}$ & 5 & $\begin{array}{l}\text { Universidad Tecnológica de } \\
\text { Toyohashi }\end{array}$ & 4 \\
\hline
\end{tabular}

Fuente: Elaboración Gerardo Tanamachi, con información del MEXT (http://www.mext.go.jp/a_menu/koutou/ shitu/1287263.htm [recuperado el 11 de julio de 2019]). 


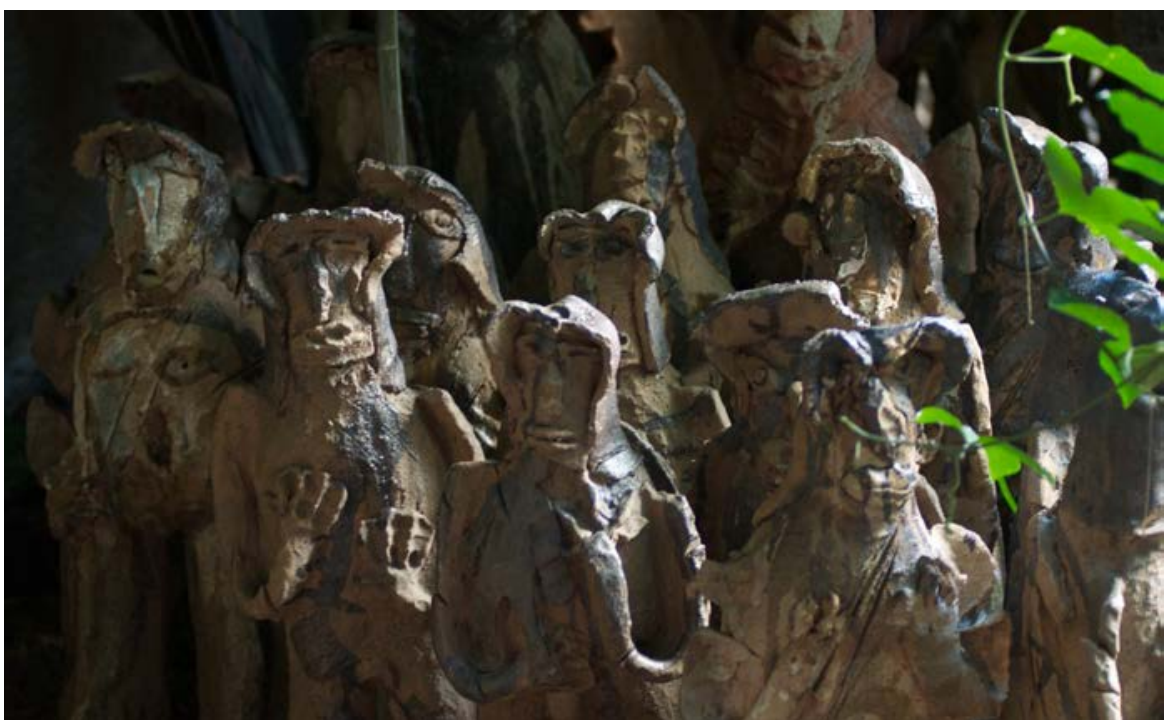

En Japón, en cambio, los acuerdos con las siete antiguas universidades imperiales (las de Tokio, Kioto, Tohoku, Kyushu, Hokkaido, Osaka y Nagoya), que gozan de singular prestigio, son apenas 12 . En la lista de las mejores universidades de Times Higher Education de 2016, esas siete universidades se ubican en los primeros diez lugares de su país, Las instituciones mexicanas que firmaron acuerdos con ellas son la UNAM, el Colmex, la Universidad Autónoma de Nuevo León (UANL), la UAG, el ITESM, la Universidad de Guadalajara, el IPN y la Universidad de Sonora. Las otras tres IES en la lista del Times son el Instituto Tecnológico de Tokio, la Universidad Médica y Dental de Tokio y la Universidad Metropolitana de Tokio. Ninguna universidad mexicana ha firmado convenios con las primeras dos. Solo el Centro de Investigación Científica de Yucatán tiene un acuerdo con la tercera. Los demás acuerdos fueron suscritos con otros tipos de instituciones públicas o con universidades privadas.

En 2009, cuando el Ministerio japonés de Educación puso en marcha el proyecto Global 30 constituyó un núcleo de 13 instituciones: siete universidades públicas ${ }^{37}$ y seis privadas: Keio, Sophia, Meiji, Waseda, Doshisha y Ritsumeikan. ${ }^{38}$ En 2016, esas 13 IES tenían 32 acuerdos vigentes con IES mexicanas (menos del $20 \%$ de los acuerdos bilaterales). Cuando el Global 30 se transformó en el Top Global University Project, abarcó 37 universidades, incluyendo 14 privadas. A 13 de las instituciones participantes se les clasificó como Top Type, por sus posibilidades de ubicarse entre las mejores instituciones del mundo o porque ya se encontraban dentro de este selecto grupo. A las demás, se les ubicó en el rubro Global Traction Type: IEs líderes en la globalización de la sociedad japonesa.

La única institución del programa Global 30 que no formó parte del nuevo proyecto fue la Universidad de Doshisha. Esta vez sí se incluyó a la Universidad de Hokkaido. ${ }^{39}$ La Tabla 2 indica que menos del $40 \%$ de los acuerdos entre instituciones mexicanas y japonesas se enmarca en dicho programa y que 19 acuerdos fueron firmados con universidades Top Type. 
Tabla 2. Instituciones participantes en el Top Global University Project

\begin{tabular}{|c|c|}
\hline Nombre de la institución & Núm. de acuerdos \\
\hline Universidad de Chiba. & 8 \\
\hline Universidad Tecnológica de Nagaoka & 7 \\
\hline Universidad Sophia $\mathbf{\Delta} \bullet$ & 7 \\
\hline Universidad Ritsumeikan $\boldsymbol{\Delta} \bullet$ & 5 \\
\hline Universidad Tecnológica de Toyohashi • & 4 \\
\hline Universidad de Kyushu $\bullet \bullet \nabla$ & 3 \\
\hline Universidad de Hiroshima $\mathbf{\square} \mathbf{\nabla}$ & 2 \\
\hline Universidad de Tokio $\boldsymbol{\bullet} \boldsymbol{\nabla}$ & 2 \\
\hline Universidad de Nagoya $\boldsymbol{\bullet \bullet \nabla}$ & 2 \\
\hline Universidad de Osaka $\boldsymbol{\bullet} \bullet \boldsymbol{\nabla}$ & 2 \\
\hline Universidad Keio $\boldsymbol{\Delta} \bullet \boldsymbol{\nabla}$ & 2 \\
\hline Universidad de Waseda $\boldsymbol{\Delta} \bullet \boldsymbol{\nabla}$ & 2 \\
\hline Universidad de Estudios Extranjeros de Tokio & 2 \\
\hline Universidad Ritsumeikan Asia Pacífico $\boldsymbol{\Delta}$ & 2 \\
\hline Universidad Meiji • & 2 \\
\hline Universidad Hosei $\boldsymbol{\Delta}$ & 2 \\
\hline Universidad Kwansei Gakuin $\boldsymbol{\Delta}$ & 2 \\
\hline Universidad de Kioto $\mathbf{\bullet} \bullet \boldsymbol{\nabla}$ & 1 \\
\hline Universidad de Tsukuba $\boldsymbol{\bullet \bullet \nabla}$ & 1 \\
\hline Universidad de Hokkaido $\mathbf{v}$ & 1 \\
\hline Universidad de Tohoku $\bullet \bullet \nabla$ & 1 \\
\hline Universidad de Kumamoto & 1 \\
\hline Instituto Tecnológico de Shibaura $\boldsymbol{\Delta}$ & 1 \\
\hline Universidad de Rikkyo (Universidad de San Pablo) $\boldsymbol{\Delta}$ & 1 \\
\hline Universidad de Kanazawa . & 1 \\
\hline Instituto Tecnológico de Tokio $\mathbf{v}$ & 0 \\
\hline Universidad Médica y Dental de Tokio $\mathbf{\nabla}$ & 0 \\
\hline Instituto Tecnológico de Kioto $\mathbf{v} \mathbf{\nabla}$ & 0 \\
\hline Universidad Soka $\Delta$ & 0 \\
\hline Universidad de las Artes de Tokio . & 0 \\
\hline Universidad de Okayama . & 0 \\
\hline Universidad de Aizu . & 0 \\
\hline Universidad Toyo $\boldsymbol{\Delta}$ & 0 \\
\hline Universidad Internacional Cristiana $\boldsymbol{\Delta}$ & 0 \\
\hline Universidad Internacional de Akita & 0 \\
\hline Universidad Internacional de Japón $\boldsymbol{\Delta}$ & 0 \\
\hline Instituto de Ciencia y Tecnología de Nara • & 0 \\
\hline Total & 64 \\
\hline
\end{tabular}

- = Universidad pública

$\Delta$ = Universidad privada

- = Universidad participante en el programa Global 30

$\mathbf{\nabla}=$ Universidad clasificada como Top Type

Fuente: Elaboración G. Tanamachi, con información del MEXT (http://www.mext.go.jp/a_menu/ koutou/kaikaku/sekaitenkai/1360288.htm [recuperado el 11 de julio de 2019]). 
No todos esos acuerdos bilaterales produjeron resultados. Según el mext, solo 100 de los 168 suscritos (menos del 60\%) auspiciaron intercambios con 794 participantes: 334 en movilidad saliente desde México hacia Japón y 460 en sentido inverso.

En 2015, como parte del Inter-University Exchange Project, el gobierno japonés empezó a financiar programas de intercambio académico con universidades de América Latina. Seis de los ocho aprobados involucraron IES mexicanas.

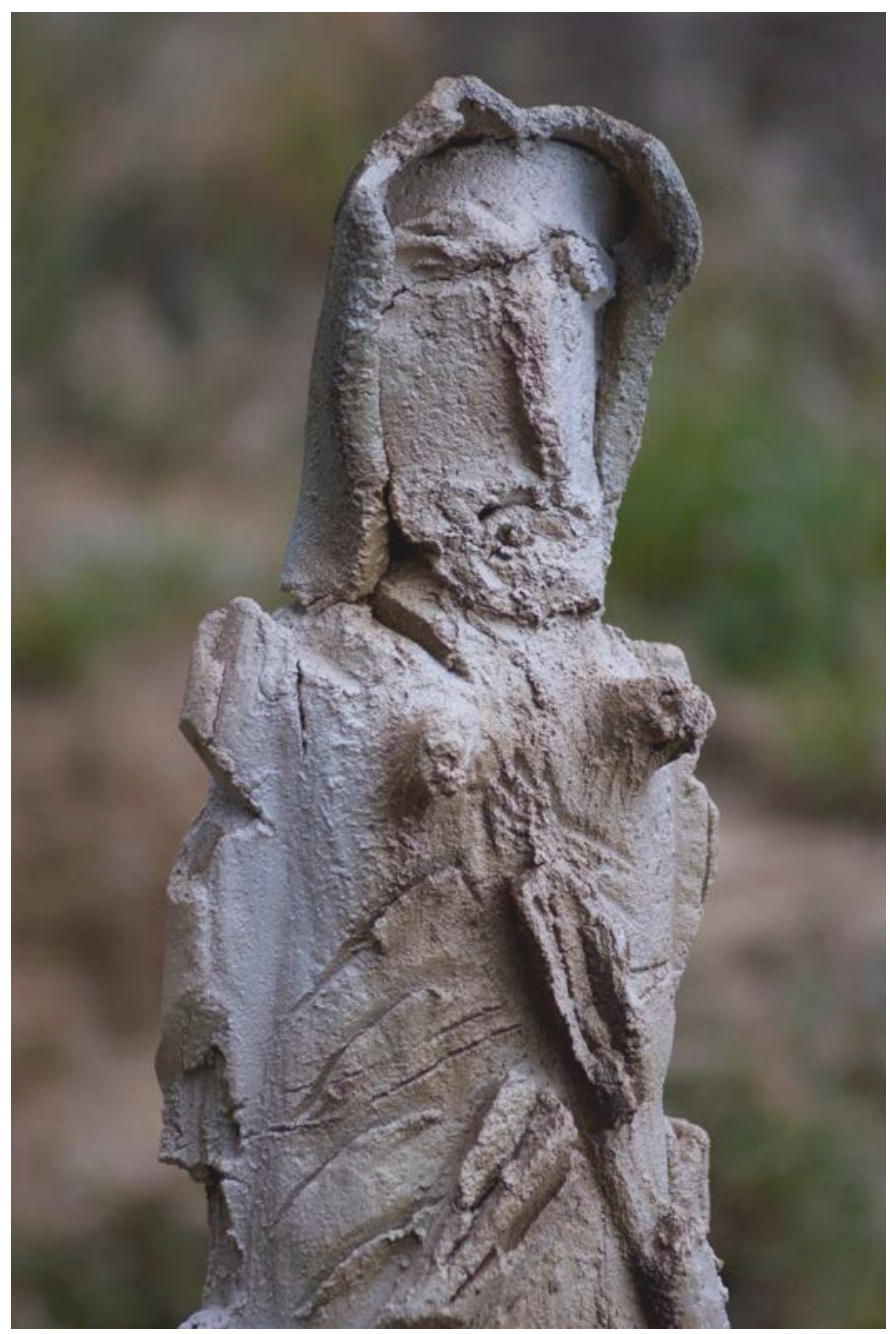


Tabla 3. Participación de IES mexicanas en el Inter-University Exchange Project

\begin{tabular}{|c|c|c|}
\hline Nombre del programa* & Instituciones japonesas involucradas & $\begin{array}{c}\text { Instituciones } \\
\text { mexicanas } \\
\text { involucradas }\end{array}$ \\
\hline $\begin{array}{l}\text { 1. Desarrollo mundial de } \\
\text { un modelo de educación } \\
\text { tecnológica a partir de los } 15 \\
\text { años de edad en colaboración } \\
\text { con México, base de } \\
\text { producción del TLCAN }\end{array}$ & $\begin{array}{l}\text { Instituto Tecnológico de Nagaoka } \\
\text { Escuela Industrial Superior } \\
\text { Especializada de Tsuruoka } \\
\text { Escuela Industrial Superior } \\
\text { Especializada de Ibaraki } \\
\text { Escuela Industrial Superior } \\
\text { Especializada de Koyama } \\
\text { Escuela Industrial Superior } \\
\text { Especializada de Nagaoka }\end{array}$ & $\begin{array}{l}\text { UGTO } \\
\text { UDEM } \\
\text { UANL }\end{array}$ \\
\hline $\begin{array}{l}\text { 2. Programa colaborativo } \\
\text { transpacífico de formación } \\
\text { de recursos humanos para } \\
\text { contribuir a una seguridad } \\
\text { social duradera }\end{array}$ & Universidad de Tsukuba & COLMEX \\
\hline $\begin{array}{l}\text { 3. Programa de innovación para } \\
\text { la vida post urbana }\end{array}$ & Universidad de Chiba & $\begin{array}{l}\text { UNAM } \\
\text { Universidad Autónoma } \\
\text { de Aguascalientes } \\
\text { Universidad } \\
\text { Veracruzana } \\
\text { UDEM } \\
\text { Universidad } \\
\text { Panamericana }\end{array}$ \\
\hline $\begin{array}{l}\text { 4. Programa colaborativo } \\
\text { de formación de recursos } \\
\text { humanos en Ciencias y } \\
\text { Humanidades para resolver los } \\
\text { problemas globales a los que } \\
\text { se enfrentan Japón y América } \\
\text { Latina }\end{array}$ & $\begin{array}{l}\text { Universidad de Tokio de Estudios } \\
\text { Extranjeros } \\
\text { Universidad de Tokio de Agricultura y } \\
\text { Tecnología } \\
\text { Universidad de Electrocomunicaciones }\end{array}$ & $\begin{array}{l}\text { Universidad Autónoma } \\
\text { Chapingo (UACh) } \\
\text { UGTO } \\
\text { UNAM } \\
\text { IPN }\end{array}$ \\
\hline $\begin{array}{l}\text { 5. Programa de intercambio } \\
\text { educativo para la solución de } \\
\text { los problemas que afectan la } \\
\text { dignidad humana y la armonía, } \\
\text { en el contexto de la migración } \\
\text { y la coexistencia }\end{array}$ & $\begin{array}{l}\text { Universidad Sophia } \\
\text { Universidad Nanzan }\end{array}$ & $\begin{array}{l}\text { UGTO } \\
\text { UNAM } \\
\text { UIA } \\
\text { Instituto Tecnológico y } \\
\text { de Estudios Superiores } \\
\text { de Occidente }\end{array}$ \\
\hline $\begin{array}{l}\text { 6. Proyecto de formación de } \\
\text { especialistas prácticos en las } \\
\text { áreas de la alimentación, la } \\
\text { agricultura y el medio ambiente } \\
\text { en América Latina }\end{array}$ & Universidad de Agricultura de Tokio & UACh \\
\hline
\end{tabular}

* Traducciones propias de los nombres en japonés de los programas.

Fuente: Elaboración G. Tanamachi, con información de la JSPS. (https://www.jsps.go.jp/j-tenkairyoku/sentei_jigyo_h27.html [recuperado el 21 de octubre de 2019]).

36 Universidades $\mid$ núm. 84, abril-junio 2020 | UDUAL $\mid$ DOI:https://doi.org/10.36888/udual.universidades.2020.84.102

Gerardo Tanamacho Castro y Sylvie Didou Aupetit | Asimetrías y consolidación progresiva de los intercambios académicos entre México y Japón, siglo XXI 


\section{Conclusiones}

Lo anterior muestra que el gobierno japonés mantiene una política pública de cooperación académica bastante estructurada, iniciada hace más de 35 años: ésta incluye la asignación de un presupuesto para apoyar proyectos específicos de internacionalización. Aunado a esto, la movilidad estudiantil de Japón es mucho mayor a la de México. Sin embargo, mientras que, en Japón, la movilidad entrante es superior a la saliente, en México sucede lo contrario.

El número de japoneses que estudian en México es comparable al de los mexicanos que estudian en Japón, lo que comprueba que México es un destino relativamente atractivo para los estudiantes japoneses. Pero, en términos cuantitativos, la movilidad estudiantil entre México y Japón es modesta, aunque Japón es un destino importante para los mexicanos que estudian en Asia y México uno relevante para los japoneses que estudian en América Latina.

Las universidades japonesas han firmado muchos acuerdos con la UNAM y el ITESM, mientras que las mexicanas han firmado relativamente pocos convenios con universidades japonesas con reconocimiento internacional o que participan en los programas gubernamentales de internacionalización de la educación superior. Las tres IES con mayor número de acuerdos con instituciones mexicanas (Chiba, Tecnológica de Nagaoka y Sophia) reciben financiamiento del Inter-University Exchange Project. Sin embargo, se ha registrado una actividad considerable en acuerdos que operaron al margen de estos mecanismos y una escasez de resultados notables, en algunos respaldados por los programas del gobierno japonés. 


\section{Notas}

1. http://www.gakkai.ne.jp/jces/kiyou.html (recuperado el 21 de octubre de 2019).

2. http://www.kyoto-u.ac.jp/ja/about/public/issue/ryugaku_tebiki (recuperado el 21 de octubre de 2019).

3. http://patlani.anuies.mx/ (recuperado el 21 de octubre de 2019).

4. http://www.rimac.mx

5. https://www.mx.emb-japan.go.jp/sp/guadalajara2014discurso.html (recuperado el 21 de octubre de 2019).

6. https://www.mx.emb-japan.go.jp/sp/linea_tiempo_3.html (recuperado el 21 de octubre de 2019).

7. https://www.mx.emb-japan.go.jp/sp/anecdota_mayo_09_2016.html (recuperado el 21 de octubre de 2019).

8 https://www.mx.emb-japan.go.jp/sp/linea_tiempo_3.html (recuperado el 21 de octubre de 2019).

9. https://embamex.sre.gob.mx/japon/index.php/es/embajada/relacion-politica/120-relacion-politica-mx-jpn/historia-de-la-relacion-bilateral/318-historia-esp (recuperado el 21 de octubre de 2019).

10. Organización intergubernamental establecida en 1950 por iniciativa de la Mancomunidad de Naciones, con el propósito de fortalecer el desarrollo económico y social de la región Asia-Pacífico.

11. https://www.aots.jp/about/ (recuperado el 21 de octubre de 2019).

12 https://embamex.sre.gob.mx/japon/index.php/es/embajada/relacion-politica/120-relacion-politica-mx-jpn/historia-de-la-relacion-bilateral/318-historia-esp (recuperado el 21 de octubre de 2019).

13. Entonces llamada Overseas Technical Cooperation Agency (OTCA). Estableció una oficina en México en 1973.

14. https://www.rimac.mx/movilidad-academica-en-la-relacion-mexicano-japonesa-en-la-posguera-programa-especial-de-intercambio-para-estudiantes-y-becarios-tecnicos-jica-conacyt-de-1971/ (recuperado el 21 de octubre de 2019).

15. https://www.gob.mx/amexcid/es/articulos/intercambio-de-estancias-tecnicas-de-alto-nivel-mx-jpn-un-puente-construido-con-la-experiencia-de-4-763-becarios?idiom=es (recuperado el 21 de octubre de 2019).

16. https://gakkai.jst.go.jp/gakkai/detail/?id=G00966 (recuperado el 21 de octubre de 2019).

17. http://www.mext.go.jp/b_menu/shingi/chukyo/chukyo4/007/gijiroku/030101/2-1.htm (recuperado el 21 de octubre de 2019).

18. https://www.fjmex.org/v2/site/nota.php?id=265\&sc=129 (recuperado el 21 de octubre de 2019).

19. Asociación propuesta por los primeros ministros de Japón y Australia, y fundada en 1980. Está conformada por comités nacionales integrados por individuos de círculos industriales, gubernamentales y académicos, y se dedica a promover la cooperación entre los países de la región Asia-Pacífico.

20. Foro multilateral establecido en 1989 para promover la integración y el crecimiento de la economía de la región Asia-Pacífico. Japón estuvo entre los países fundadores.

21. https://www.oecd.org/centrodemexico/15aosdemexicoenlaocde.htm (recuperado el 21 de octubre de 2019).

22. Organización intergubernamental con el propósito de fomentar el comercio y el desarrollo económico. Japón era miembro desde 1964.

23. https://www.studyjapan.go.jp/jp/toj/toj09j.html (recuperado el 21 de octubre de 2019).

24. http://www.mext.go.jp/component/a_menu/education/detail/__icsFiles/afieldfile/2017/03/30/1383779_06.pdf (recuperado el 21 de octubre de 2019).

25 https://www.jsps.go.jp/j-tenkairyoku/ (recuperado el 21 de octubre de 2019).

26. https://www.jsps.go.jp/aboutus/index2.html\#enkaku (recuperado el 21 de octubre de 2019).

27. https://www.jsps.go.jp/j-gjinzai/news/news.html (recuperado el 21 de octubre de 2019). 
28. http://www.mext.go.jp/b_menu/houdou/26/09/1352218.htm (recuperado el 21 de octubre de 2019).

29. https://www.jsps.go.jp/english/e-tgu/selection.html (recuperado el 21 de octubre de 2019).

30. https://www.gob.mx/se/prensa/mexico-y-japon-se-comprometen-a-continuar-el-mejoramiento-de-su-ambiente-de-negocios (recuperado el 21 de octubre de 2019).

31. https://www.mx.emb-japan.go.jp/rectores2014sp.pdf (recuperado el 21 de octubre de 2019).

32. https://www.rimac.mx/las-primeras-dos-cumbres-de-rectores-mexico-japon-un-fomento-a-la-cooperacion-academica-bilateral/ (recuperado el 21 de octubre de 2019).

33. http://www.rimac.mx/la-tercera-cumbre-de-rectores-mexico-japon-un-balance-preliminar-de-resultados/ (recuperado el 21 de octubre de 2019).

34. https://www.mx.emb-japan.go.jp/itprtop_es/00_001873.html (recuperado el 21 de octubre de 2019).

35. Bajo la categoría de América Central y del Sur, en la cual se incluye a México.

36. http://www.mext.go.jp/a_menu/koutou/shitu/1287263.htm (recuperado el 11 de julio de 2019).

37. Se incluyó a las antiguas universidades imperiales, a excepción de Hokkaido con la cual el ITESM tiene un acuerdo. La séptima fue la Universidad de Tsukuba, institución con la que, en México, solo el COLMEX tiene un convenio.

38. http://www.mext.go.jp/a_menu/koutou/kaikaku/1383342.htm (recuperado el 11 de julio de 2019).

39. http://www.mext.go.jp/a_menu/koutou/kaikaku/sekaitenkai/1360288.htm (recuperado el 11 de julio de 2019).

\section{Referencias}

Abe, Hitoshi, et al. (2018). Gurōbaru Kankyō de Hagukumu Yottsu no Chikara: Ryūgaku Zengo ni Okeru Haken Gakusei no Konpitenshī Henka ni tsuite (Las cuatro capacidades que se deben cultivar en un ambiente global: acerca de los cambios en las competencias de los estudiantes enviados al extranjero antes y después de sus estudios en otros países). Hitotsubashi Daigaku Kokusai Kyōiku Sentā Kiyō, 9: 5-18.

Barajas, Mónica (2002). La cooperación técnica y educativa-cultural entre México y Japón de 1970 a 1994. Tesina profesional. Universidad Autónoma Metropolitana, Unidad Iztapalapa.

Castañeda, Erica (2000). Las relaciones culturales-educativas entre México y Japón: 1976-1994. Tesina profesional. Universidad Autónoma Metropolitana, Unidad Iztapalapa.

Comparative \& International Education Society (2015). CIES Bibliography 2014. Comparative Education Review, 59, S4: S1-S228.

Didou, Sylvie (1998). Cooperación científica y tecnológica japonesa en México. Comercio Exterior, 48(2): 114-121.

Didou, Sylvie (2019). La internacionalización de las universidades mexicanas hacia Asia del Este. ¿Una modalidad de cooperación Sur-Sur? Perfiles Educativos, XLI(163): 159-175.

Embajada del Japón en México (2016). Boletín informativo de la Embajada del Japón, 3. Embajada del Japón en México.

García, David Horacio y Sepúlveda, Patricia Rebeca (2016). Factores determinantes para los estudiantes internacionales en la selección de una universidad destino a través de la revisión de literatura: un preámbulo para el estudio de los estudiantes internacionales en México. Política, Globalidad y Ciudadanía, 2(3): 13-35.

Granados, Ulises (2016). La relación México-Japón: más allá de la coyuntura económica. Revista Mexicana de Política Exterior, 108: 69-85.

JASSO (2019a). Heisei 30 Nendo Gaikokujin Ryūgakusei Zaiseki Jōkyō Chōsa Kekka (Resultados del estudio sobre la situación de los estudiantes extranjeros matriculados en Japón en 2018). JASSO.

JASSO (2019b). Heisei 29 Nendo Kyōtei ni Motozuku Nihonjin Ryūgaku Jōkyō Chōsa Kekka (Resultados del estudio sobre la situación de los estudiantes japoneses matriculados en el extranjero en 2017). JASSO. 
Kerber, Víctor (2008). El sol naciente de Vicente Fox. México y Japón 2000-2006. Foro Internacional, XLIII(1-2): 352-374.

MEXT (2019). “Gaikokujin Ryūgakusei Zaiseki Jōkyō Chōsa” Oyobi "Nihonjin no Kaigai Ryūgakushasū” nado ni tsuite (Acerca del "Estudio sobre la situación de los estudiantes extranjeros matriculados en Japón", el "Número de estudiantes japoneses en el extranjero", etc.). MEXT.

Ochiai, Kazuyasu (2019). Transferencia intercultural de conocimientos: estrategia formal y comunicación sustantiva. En Didou, Sylvie, França, Thais y Padilla, Beatriz (eds.). Geoestrategia de la internacionalización y espacialidad de las migraciones académicas. UDUAL-CINVESTAV.

Ota, Hiroshi (2011). Naze Kaigai Ryūgaku Banare wa Okotte iru no ka (¿Por qué se ha presentado un distanciamiento de los estudios en el extranjero?). Kyōiku to Igaku, 59(1): 68-76.

Ota, Hiroshi (2012). Changes in Internationalization of Japanese Higher Education. IAU Horizons, 17: 26-27.

Ota, Hiroshi y Watabe, Yuki (2018). Mapping Internationalization of Japanese Universities: Goals, Strategies, and Indicators. En International Briefs for Higher Education Leaders, 7. American Council on Education y The Boston College Center for International Higher Education.

Ota Mishima, María Elena (1975). Ryuji Oki: Director, Japonés para hispanohablantes (reseña). Estudios de Asia y África, 3(29): 363-367.

Ota Mishima, María Elena (1983). Siete Migraciones Japonesas en México, 1890-1978. El Colegio de México.

Quiroz, Elena (2013). Internacionalización e interculturalidad; un reto para la educación superior. Reencuentro, 67: 59-64.

Ramírez, Juan José (coordinador) (2019). La internacionalización de las Instituciones de Educación Superior privadas mexicanas. CINVESTAV/RIMAC/UNESCO.

Ramírez, Juan José (coordinador) (2017). La internacionalización en las Instituciones de Educación Superior mexicanas. Experiencias de vinculación con Asia del Pacífico. RIMAC/CONACYT/CINVESTAV.

Rodríguez, Juan David (2013). Educación universitaria en Japón: políticas para la atracción de estudiantes extranjeros. Mundo Asia Pacífico, 2(3): 59-66.

Shimauchi, Sae (2014). Gurōbaru Jinzai Ikusei to Daigaku no Kokusaika ni Kansuru Ikkōsatsu. Yokohama Shiritsu Daigaku Ronsō Jinbun Kagaku Keiretsu, 66(1): 109-126.

Shinmi, Yukiko y Ota, Hiroshi (2018). "Super-Short-Term” Study Abroad in Japan: A Dramatic Increase. International Higher Education, 94: 13-15.

Sugimura, Miki (2015). Roles of Language in Multicultural Education in the Context of Internationalisation. Education Studies in Japan: International Yearbook, 9: 3-15.

Tanaka, Kyoko (2010). Nihon Ryūgaku 30 Nengo -Raten Amerika Shusshinsha no Jirei Kenkyū- (30 años después de estudiar en Japón. El caso de la investigación sobre los latinoamericanos). Hiroshima Daigaku Ryūgakusei Kyōiku.

Toda, Makoto (traductor). Historia de las Relaciones Mexicano-Japonesas (traducción del Nichiboku Kōryūshi), tomos I (2012) y II (2013). Artes Gráficas Panorama.

Tsuboi, Tsuyoshi (2005). Ryūgakusei Chōsa kara Mieru Mono -Komadai Ryūgakusei 15 Nenkan no Dōkō(Lo que se aprecia en las investigaciones sobre los estudiantes en el extranjero. Las tendencias durante 15 años de los estudiantes de la Universidad Komazawa en el extranjero). Komazawa Daigaku Bungakubu Kenkyū Kiyō, 64: 1-17.

Tsuboi, Tsuyoshi (2006). Ryūgakusei Kenkyū Kanren Bunken Mokroku (1955-2005) (Catálogo de bibliografía relacionada con las investigaciones sobre los estudiantes en el extranjero, 1955-2005). Komazawa Shakaigaku Kenkyū, 64: 23-65.

Uscanga, Carlos (2011a). México y Japón en los años treinta: los avatares del intercambio académico. Revista Relaciones Internacionales de la UNAM, 110: 159-171.

Uscanga, Carlos (2011b). La cooperación triangular México-Japón. Revista Española de Desarrollo y Cooperación, 28: 83-93.

Vavrus, Frances \& Pekol, Amy (2015). Critical Internationalization: Moving from Theory to Practice. FIRE: Forum for International Research in Education, (2) 2: 5-21.

Yonezawa, Akiyoshi (2010). Much Ado About Ranking: Why Can’t Japanese Universities Internationalize? Japan Forum, 22 (1-2): 121-137.

40 Universidades $\mid$ núm. 84, abril-junio 2020 $\mid$ UDUAL $\mid$ DOI:https://doi.org/10.36888/udual.universidades.2020.84.102 